\title{
Study of Quantitative Traits and Association for Yield and its Components in Black Gram (Vigna mungo (L.) Hepper) Genotypes
}

\author{
Alle N. Sarath C. Reddy*, Gabrial M. Lal and V. Siva Krishna \\ Department of Genetics and Plant Breeding, Naini Agriculture Institute, Sam Higginbottom \\ University of Agriculture, Technology and Sciences, Prayagraj, Uttar Pradesh, India \\ *Corresponding author
}

\begin{tabular}{l} 
K e y w o r d s \\
Black gram [Vigna \\
mungo (L.) \\
Hepper], GCV, \\
PCV, Variability, \\
Heritability, \\
Genetic Advance, \\
Correlation and \\
Path analysis \\
Article Info \\
\hline $\begin{array}{l}\text { Accepted: } \\
\text { 15 August } 2020 \\
\text { Available Online: } \\
10 \text { September } 2020\end{array}$ \\
\hline
\end{tabular}

\section{Keywords}

Black gram [Vigna mungo (L.)

Hepper], GCV, PCV, Variability,

Heritability,

Genetic Advance,

Correlation and

Accepted:

Available Online:

\section{A B S T R A C T}

The present investigation was undertaken in 23 genotypes of blackgram [Vigna mungo (L.) Hepper] to study the genetic variability parameters, correlation and path analysis during Kharif, 2019 in Randomized Block Design with three replications. The observations were recorded for fourteen quantitative characters to study genetic variability, heritability, genetic advance, correlation and path analysis. The analysis of variance indicated significant differences among twenty three genotypes for all the characters studied. On the basis of mean performance, the highest seed yield per plant was observed for the blackgram genotypes SU-Urd-23 followed by VBN-6, SU-Urd-34 and SU-Urd-33.The estimates of PCV for all characters were higher than the estimates of GCV indicating the presence of environmental component along with genetic component. High GCV and PCV were observed for number of clusters per plant, number of pods per plant, harvest index, seed yield per plant indicating high variability for these traits. High heritability coupled with high genetic advance as per cent of mean was recorded for plant height, number of clusters per plant, number of pods per plant and harvest index. This indicates the preponderance of additive gene action and hence phenotypic selection would be more effective for further improvement of these traits. Correlation association studies revealed that the characters number of clusters per plant, number of pods per plant, number of pods per cluster, number of seeds per pod and harvest index showed significant and positive correlation with seed yield per plant indicating that selection based on these characters would bring about simultaneous improvement in the seed yield. Path analysis revealed that number of clusters per plant, number of pods per plant, number of pods per cluster and harvest index hadtrue relationship by establishing significant positive associations and positive direct effects on seed yield per plant.

\section{Introduction}

Blackgram [Vigna mungo (L.) Hepper] is an annual leguminous crop belongs to family Fabaceae and sub-family Papilionaceae with a chromosome number of 2n=22 (Dana, 1980) and originated from Indian continent (De Candolle, 1882, Vavilov, 1926 and Zukovskij, 1962). It is popularly known as "urdbean or mash. It is an important self-pollinating 
diploid grain legume (Naga et al., 2006) domesticated from $V$. mungo var. silvestris (Lukoki et al., 1980).

Black gram is an economically important grain legume crop in Asia, widely cultivated on marginal lands with low inputs during Kharif, Rabi and Zaid seasons. A seed of blackgram is the most important product which is consumed in the form of dal (whole or split, husked and unhusked) or parched. Many South Indian dishes as dosa and idli have blackgram as main ingredient. The urd flour is also used in making papad and dahivada.

Pulses are the chief and cheapest sources of plant-based dietary protein for the vegetarian people. Protein content in pulses ranges from $20-40 \%$ as compared to $8-12 \%$ of cereals. Moreover, they are an excellent source of lysine, an essential amino acid with an average of $65 \pm 7 \mathrm{mg} / \mathrm{g}$ as compared to $29 \pm 7$ $\mathrm{mg} / \mathrm{g}$ in cereals and fairly supplies a good quantity of vitamins (thiamine, riboflavin, niacin and vitamin B6) and much needed minerals viz., iron, zinc, calcium and phosphorus

India is the largest producer and consumer of blackgram, with an area about 50.31 lakhs hectares, production of 32.84 lakhs tons and productivity of $653 \mathrm{~kg} \mathrm{ha}^{-1}$. In Uttar Pradesh, it is grown in an area about 5.88 lakh hectares with a production of 3.05 lakh tons and a productivity of $520 \mathrm{~kg} \mathrm{ha}^{-1}$ (Directorate Pulse Development, Ministry of Agriculture and Farmers Welfare Annual Report (2018-19)).

Seed yield is a complex trait and is the resultant product of various morphological, physiological and biological components. Hence it is important to know the genetic architecture and nature of gene action governing yield and its component traits to increase the yield per unit area. At the study of inheritance of these traits through the estimation of different genetic parameters like components of variances, genotypic and phenotypic coefficients of variability, heritability and genetic advance is helpful for framing an effective breeding programme.

\section{Materials and Methods}

The experiment was carried out in the Field Experimentation Centre of Department of Genetics and Plant Breeding, Naini Agricultural Institute, Sam Higginbottom University of Agriculture, Technology and Sciences, Prayagraj, U.P, India. The experimental materials for the present study consisted of 23 genotypes of black gram. The experiment was laid out in a Randomized Block Design (RBD) with three replications. The experimental material was planted in three replications. Each replication consisted of 23 genotypes randomized and replicated within each block.

The observations were recorded for fourteen quantitative characters viz. days to $50 \%$ flowering, days to $50 \%$ pod setting, days to maturity, plant height, number of primary branches per plant, number of clusters per plant, number of pods per plant, number of pods per clusters, number of seeds per pod, pod length, 100 seed weight, biological yield per plant, harvest index, seed yield per plant.

Mean values were computed and data were analysed for analysis of variance as suggested Fisher (1936) given in table 1. Phenotypic coefficient of variation (PCV) and genotypic coefficient of variation (GCV) were given by Burton (1952). Heritability in broad sense was given by Lush (1949) and Burton and Devane (1953). Genetic advance was given by Lush (1949) and Johnson et al., (1955). Correlation coefficients are estimated as suggested by $\mathrm{Al}$ Jibouri et al., (1958) and the path analysis was calculated as suggested by Dewey and Lu (1959). 


\section{Results and Discussion}

The analysis of variance carried out 14 quantitative characters revealed significant differences among the genotypes for all the characters (Table 1). Hence it was concluded that there is scope for ample genetic variation among the genotypes under study and further statistical analysis was carried out. On the basis of mean performance, the highest seed yield per plant was observed for the blackgram genotypes SU-Urd-23 followed by VBN-6, SU-Urd-34 and SU-Urd-33.

The PCV was higher than GCV for all the characters under study which indicated that the environmental factors influencing the characters studied. High GCV was recorded for number of clusters per plant (30.944), number of pods per plant (23.207) and harvest index (22.023). Moderate GCV was recorded for plant height (19.89), number of branches per plant (12.762), number of pods per cluster (13.343) and seed yield per plant (16.971). Low GCV was recorded was recorded for days to $50 \%$ flowering (7.929), days to $50 \%$ Pods Setting (7.191), days to maturity (5.608), number of seeds per pod (5.753), pod length (4.734), 100 seed weight (5.429) and biological yield per plant (8.417) (Table 2).

The phenotypic coefficient of variation (PCV) ranged from $5.732 \%$ for 100 seed weight to $33.056 \%$ number of clusters per plant. The PCV estimates would show that the phenotypic variability was low (below 10\%) for days to $50 \%$ flowering (8.711), days to $50 \%$ Pods Setting (7.848), days to maturity (6.178), number of seeds per pod (8.019), pod length (8.273), 100 seed weight (5.732) and biological yield per plant (9.636). High PCV (>20\%) for plant height (20.277), number of branches per plant (21.569), number of clusters per plant (33.056), number of pods per plant (26.615) number of pods per cluster (22.781), harvest index (25.61) and seed yield per plant (24.528) (Table 2). High phenotypic and genotypic coefficients of variability were observed for number of clusters per plant, number of pods per plant, harvest index, seed yield per plant indicating high variability for these traits. Moderate to low coefficients of variability was noticed for remaining traits revealing the presence of moderate variability.

The estimates of heritability (\%) in the broad sense for 14 characters studied, which range from 32.74 to $96.222 \%$. High heritability (broad sense) was recorded for characters i.e., plant height (96.222\%), days to $50 \%$ flowering (82.855), days to 50\% Pods Setting (83.959), days to maturity (82.376), number of clusters per plant (87.631), number of pods per plant (76.03), 100 seed weight (89.691), biological yield per plant (76.297) and Harvest index (73.948). Moderate heritability (broad sense) was recorded for character number of branches per plant (35.011), number of pods per cluster (34.307), number of seeds per pod (51.471), pod length (32.74) and seed yield per plant (47.871).

In the present study, the GA as \% of mean ranged from $5.58 \%$ (pod length) to $59.673 \%$ (number of clusters per plant). It was low (below 10\%) for plant height (5.58\%), number of seeds per pod (8.503) and moderate GA as \% of mean (10-20) for days to $50 \%$ flowering (14.867), days to $50 \%$ Pods Setting (13.574), days to maturity (10.484), number of branches per plant (15.556), number of pods per cluster (16.1), 100 seed weight (10.591), biological yield per plant (15.146). While high GA as \% of mean (above 20\%) recorded in plant height (40.192), number of clusters per plant (59.673), number of pods per plant (41.685), harvest index (39.012), seed yield per plant (24.188). High heritability coupled with high genetic advance as per cent of mean was recorded for plant height, number of clusters 
per plant, number of pods per plant and harvest index which is indicative of the fact that, there is lesser influence of the environment in expression of these characters and prevalence of additive gene action in their inheritance, hence they are amenable for simple selection. A high genetic gain can be achieved from phenotypic selection.

Genotypic correlation between seed yield per plant and other characters showed the positive significant genotypic association with number of clusters per plant $(0.820 * *)$, number of pods per plant $(0.750 * *)$, number of pods per cluster $\left(0.650^{* *}\right)$, number of seeds per pod $\left(0.470^{* *}\right)$ and harvest index $\left(0.857^{* *}\right)$.

Phenotypic correlation between seed yield per plant and other characters showed the positive significant genotypic association with number of clusters per plant $\left(0.665^{* *}\right)$, number of pods per plant $(0.719 * *)$, number of pods per cluster $\left(0.338^{* *}\right)$, number of seeds per pod $(0.358 * *)$, pod length $(0.419 * *)$ and harvest index $(0.798 * *)$ (Table 3-5).

Table.1 Analysis of variance for seed yield and its components in Black gram

\begin{tabular}{|l|c|c|c|}
\hline \multicolumn{1}{|c|}{ Characters } & Replication & Treatment & Error \\
\cline { 2 - 4 } & $(\mathrm{df}=2)$ & $(\mathrm{df}=22)$ & $(\mathrm{df}=48)$ \\
\hline Days to 50\% flowering & 5.261 & $39.03 * *$ & 2.518 \\
\hline Days to 50\% Pods Setting & 6.971 & $46.838^{* *}$ & 2.804 \\
\hline Days to maturity & 6.971 & $44.858^{* *}$ & 2.986 \\
\hline Plant height (cm) & 0.884 & $271.399 * *$ & 3.509 \\
\hline Number of branches per plant & 0.4805 & $0.355^{* *}$ & 0.136 \\
\hline Number of clusters per plant & 1.8965 & $69.981 * *$ & 3.145 \\
\hline Number of pods per plant & 0.0395 & $101.557 * *$ & 9.658 \\
\hline Number of pods per cluster & 0.689 & $0.305 * *$ & 0.119 \\
\hline Number of seeds per pod & 0.086 & $0.44 * *$ & 0.105 \\
\hline Pod length (cm) & 0.836 & $0.161 * *$ & 0.065 \\
\hline 100 seed weight (g) & 0.0065 & $0.143 * *$ & 0.005 \\
\hline Biological yield/plant (g) & 0.2655 & $6.541^{* *}$ & 0.614 \\
\hline Harvest index (\%) & 2.046 & $190.227 * *$ & 19.991 \\
\hline Seed yield/plant (g) & 0.126 & $3.906 * *$ & 1.04 \\
\hline
\end{tabular}

** Significance at $1 \%$ level 
Table.2 Genetic parameters for 14 characters of 23 blackgram genotypes

\begin{tabular}{|l|c|c|c|c|c|c|}
\hline \multicolumn{1}{|c|}{ Characters } & Grand Mean & $\begin{array}{c}\text { GCV } \\
(\%)\end{array}$ & $\begin{array}{c}\text { PCV } \\
(\%)\end{array}$ & $\begin{array}{c}\text { Heritability } \\
(\%)\end{array}$ & $\begin{array}{c}\text { Genetic } \\
\text { Advance }\end{array}$ & $\begin{array}{c}\text { Genetic } \\
\text { Advance } \\
\text { as \% of } \\
\text { Means }\end{array}$ \\
\hline Days to 50\% flowering & 44.00 & 7.929 & 8.711 & 82.855 & 6.542 & 14.867 \\
\hline Days to 50\% Pods Setting & 53.28 & 7.191 & 7.848 & 83.959 & 7.232 & 13.574 \\
\hline Days to maturity & 66.62 & 5.608 & 6.178 & 82.376 & 6.985 & 10.484 \\
\hline Plant height (cm) & 47.51 & 19.89 & 20.277 & 96.222 & 19.095 & 40.192 \\
\hline $\begin{array}{l}\text { Number of branches per } \\
\text { plant }\end{array}$ & 2.12 & 12.762 & 21.569 & 35.011 & 0.33 & 15.556 \\
\hline $\begin{array}{l}\text { Number of clusters per } \\
\text { plant }\end{array}$ & 15.25 & 30.944 & 33.056 & 87.631 & 9.102 & 59.673 \\
\hline Number of pods per plant & 23.85 & 23.207 & 26.615 & 76.03 & 9.942 & 41.685 \\
\hline Number of pods per cluster & 1.87 & 13.343 & 22.781 & 34.307 & 0.301 & 16.1 \\
\hline Number of seeds per pod & 5.81 & 5.753 & 8.019 & 51.471 & 0.494 & 8.503 \\
\hline Pod length (cm) & 3.77 & 4.734 & 8.273 & 32.74 & 0.21 & 5.58 \\
\hline 100 seed weight (g) & 3.948 & 5.429 & 5.732 & 89.691 & 0.418 & 10.591 \\
\hline Biological yield/plant (g) & 16.699 & 8.417 & 9.636 & 76.297 & 2.529 & 15.146 \\
\hline Harvest index (\%) & 34.205 & 22.023 & 25.61 & 73.948 & 13.344 & 39.012 \\
\hline Seed yield/plant (g) & 5.759 & 16.971 & 24.528 & 47.871 & 1.393 & 24.188 \\
\hline
\end{tabular}


Table.3 Genotypic and phenotypic correlation coefficients of Black gram genotypes

\begin{tabular}{|c|c|c|c|c|c|c|c|c|c|c|c|c|c|c|}
\hline Characters & & DP50 & DM & $\mathbf{P H}$ & PB & CPP & PPP & PPC & SPP & PL & HSW & BY & HI & GY \\
\hline \multirow{2}{*}{$\begin{array}{l}\text { Days to } 50 \% \\
\text { flowering }\end{array}$} & $\mathrm{G}$ & $0.790^{* *}$ & $0.786^{* *}$ & $0.214^{\mathrm{NS}}$ & $0.054^{\mathrm{NS}}$ & $-0.324^{* *}$ & $-0.191^{\mathrm{NS}}$ & $-0.699^{* *}$ & $0.506^{* *}$ & $-0.085^{\mathrm{NS}}$ & $-0.183^{\mathrm{NS}}$ & $0.147^{\mathrm{NS}}$ & $-0.143^{\mathrm{NS}}$ & $-0.125^{\mathrm{NS}}$ \\
\hline & $\mathrm{P}$ & $0.808^{* *}$ & $0.798^{* *}$ & $0.205^{\mathrm{NS}}$ & $0.007^{\mathrm{NS}}$ & $-0.268^{*}$ & $-0.129^{\mathrm{NS}}$ & $-0.382^{* *}$ & $0.285^{*}$ & $0.010^{\mathrm{NS}}$ & $-0.144^{\mathrm{NS}}$ & $0.104^{\mathrm{NS}}$ & $-0.109^{\mathrm{NS}}$ & $-0.073^{\mathrm{NS}}$ \\
\hline \multirow{2}{*}{$\begin{array}{l}\text { Days to } 50 \% \\
\text { Pods Setting }\end{array}$} & $\mathrm{G}$ & & $0.901^{* *}$ & $-0.159^{\mathrm{NS}}$ & $-0.108^{\mathrm{NS}}$ & $-0.249^{*}$ & $-0.150^{\mathrm{NS}}$ & $-0.609^{* *}$ & $0.562^{* *}$ & $0.363^{* *}$ & $-0.232^{\mathrm{NS}}$ & $-0.217^{\mathrm{NS}}$ & $0.157^{\mathrm{NS}}$ & $0.060^{\mathrm{NS}}$ \\
\hline & $\mathrm{P}$ & & $0.993^{* *}$ & $-0.130^{\mathrm{NS}}$ & $-0.060^{\mathrm{NS}}$ & $-0.208^{\mathrm{NS}}$ & $-0.099^{\mathrm{NS}}$ & $-0.362^{* *}$ & $0.330^{* * *}$ & $0.272^{*}$ & $-0.184^{\mathrm{NS}}$ & $-0.171^{\mathrm{NS}}$ & $0.139^{\mathrm{NS}}$ & $0.057^{\mathrm{NS}}$ \\
\hline \multirow{2}{*}{$\begin{array}{l}\text { Days to } \\
\text { maturity }\end{array}$} & $\mathrm{G}$ & & & $-0.158^{\mathrm{NS}}$ & $-0.149^{\mathrm{NS}}$ & $-0.237^{*}$ & $-0.143^{\mathrm{NS}}$ & $-0.597^{* *}$ & $0.521^{* *}$ & $0.344^{* *}$ & $-0.245^{*}$ & $-0.230^{\mathrm{NS}}$ & $0.164^{\mathrm{NS}}$ & $0.064^{\mathrm{NS}}$ \\
\hline & $\mathrm{P}$ & & & $-0.130^{\mathrm{NS}}$ & $-0.066^{\mathrm{NS}}$ & $-0.192^{\mathrm{NS}}$ & $-0.087^{\mathrm{NS}}$ & $-0.338^{* *}$ & $0.318^{* *}$ & $0.266^{*}$ & $-0.186^{\mathrm{NS}}$ & $-0.175^{\mathrm{NS}}$ & $0.143^{\mathrm{NS}}$ & $0.071^{\mathrm{NS}}$ \\
\hline $\begin{array}{l}\text { Plant height } \\
\text { (cm) }\end{array}$ & $\mathrm{P}$ & & & & $-0.240^{*}$ & $0.135^{\mathrm{NS}}$ & $0.127^{\mathrm{NS}}$ & $0.063^{\mathrm{NS}}$ & $-0.178^{\mathrm{NS}}$ & $-0.270^{*}$ & $-0.311^{* *}$ & $0.736^{* *}$ & $-0.300^{*}$ & $0.048^{\mathrm{NS}}$ \\
\hline \multirow{2}{*}{$\begin{array}{l}\text { Number of } \\
\text { branches/plant }\end{array}$} & $\mathrm{G}$ & & & & & $-0.223^{\mathrm{NS}}$ & $-0.441^{* *}$ & $-0.908^{* *}$ & $0.072^{\mathrm{NS}}$ & $0.269^{*}$ & $0.532^{* *}$ & $-0.400^{* *}$ & $-0.070^{\mathrm{NS}}$ & $-0.454^{* *}$ \\
\hline & $\mathrm{P}$ & & & & & $-0.062^{\mathrm{NS}}$ & $-0.133^{\mathrm{NS}}$ & $-0.225^{\mathrm{NS}}$ & $0.199^{\mathrm{NS}}$ & $-0.025^{\mathrm{NS}}$ & $0.329^{* *}$ & $-0.203^{\mathrm{NS}}$ & $0.042^{\mathrm{NS}}$ & $-0.057^{\mathrm{NS}}$ \\
\hline \multirow{2}{*}{$\begin{array}{l}\text { Number of } \\
\text { clusters/plant }\end{array}$} & $\mathrm{G}$ & & & & & & $0.449^{* *}$ & $0.455^{* *}$ & $-0.141^{\mathrm{NS}}$ & $-0.185^{\mathrm{NS}}$ & $-0.272^{*}$ & $0.010^{\mathrm{NS}}$ & $0.726^{* *}$ & $0.820^{* *}$ \\
\hline & $\mathrm{P}$ & & & & & & $0.477^{* *}$ & $0.320^{* *}$ & $-0.076^{\mathrm{NS}}$ & $-0.097^{\mathrm{NS}}$ & $-0.234^{\mathrm{NS}}$ & $-0.039^{\mathrm{NS}}$ & $0.678^{* *}$ & $0.665^{* *}$ \\
\hline $\begin{array}{l}\text { Number of } \\
\text { pods/plant }\end{array}$ & $\mathrm{G}$ & & & & & & & $0.392^{* *}$ & $-0.133^{\mathrm{NS}}$ & $-0.104^{\mathrm{NS}}$ & $-0.439^{* *}$ & $0.316^{* *}$ & $0.470^{* *}$ & $0.750^{* *}$ \\
\hline \multirow{2}{*}{$\begin{array}{l}\text { Number of } \\
\text { pods/cluster }\end{array}$} & $\mathrm{G}$ & & & & & & & & $-0.622^{* *}$ & $-0.694^{* *}$ & $-0.220^{\mathrm{NS}}$ & $0.181^{\mathrm{NS}}$ & $0.033^{\mathrm{NS}}$ & $0.650^{* *}$ \\
\hline & $\mathrm{P}$ & & & & & & & & $-0.202^{\mathrm{NS}}$ & $-0.318^{* *}$ & $-0.059^{\mathrm{NS}}$ & $0.034^{\mathrm{NS}}$ & $0.133^{\mathrm{NS}}$ & $0.338^{* *}$ \\
\hline \multirow{2}{*}{$\begin{array}{l}\text { Number of } \\
\text { seeds/pod }\end{array}$} & $\mathrm{G}$ & & & & & & & & & $0.552^{* *}$ & $-0.123^{\mathrm{NS}}$ & $-0.242^{*}$ & $0.259^{*}$ & $0.470^{* *}$ \\
\hline & $\mathrm{P}$ & & & & & & & & & $0.111^{\mathrm{NS}}$ & $-0.071^{\mathrm{NS}}$ & $-0.147^{\mathrm{NS}}$ & $0.192^{\mathrm{NS}}$ & $0.358^{* *}$ \\
\hline \multirow[t]{2}{*}{ Pod length (cm) } & $\mathrm{G}$ & & & & & & & & & & $-0.052^{\mathrm{NS}}$ & $-0.345^{* *}$ & $0.320^{* *}$ & $-0.374 * *$ \\
\hline & $\mathrm{P}$ & & & & & & & & & & $-0.080^{\mathrm{NS}}$ & $-0.217^{\mathrm{NS}}$ & $0.158^{\mathrm{NS}}$ & $0.419^{* *}$ \\
\hline \multirow{2}{*}{$\begin{array}{l}100 \text { seed weight } \\
\text { (g) }\end{array}$} & $\mathrm{G}$ & & & & & & & & & & & $-0.569^{* *}$ & $-0.098^{\mathrm{NS}}$ & $-0.387^{* *}$ \\
\hline & $\mathrm{P}$ & & & & & & & & & & & $-0.467^{* *}$ & $-0.030^{\mathrm{NS}}$ & $-0.198^{\mathrm{NS}}$ \\
\hline \multirow{2}{*}{$\begin{array}{l}\text { Biological } \\
\text { yield/plant (g) }\end{array}$} & $\mathrm{G}$ & & & & & & & & & & & & $-0.404^{* *}$ & $0.072^{\mathrm{NS}}$ \\
\hline & $\mathrm{P}$ & & & & & & & & & & & & $-0.385^{* *}$ & $0.003^{\mathrm{NS}}$ \\
\hline \multirow{2}{*}{$\begin{array}{l}\text { Harvest index } \\
(\%)\end{array}$} & $\mathrm{G}$ & & & & & & & & & & & & & $0.857^{* *}$ \\
\hline & $\mathrm{P}$ & & & & & & & & & & & & & $0.798^{* *}$ \\
\hline
\end{tabular}


Table.4 Direct and indirect effects between yield and its related traits in 23 Black gram genotypes at genotypic level

\begin{tabular}{|c|c|c|c|c|c|c|c|c|c|c|c|c|c|}
\hline Characters & DF50 & DP50 & DM & PH & PB & CPP & PPP & PPC & SPP & PL & HSW & BY & HI \\
\hline $\begin{array}{c}\text { Days to } 50 \% \\
\text { flowering }\end{array}$ & 0.39691 & 0.73649 & -0.62072 & 0.00384 & 0.0033 & -0.12971 & -0.06164 & -0.2816 & -0.06778 & -0.0281 & -0.04422 & 0.04338 & -0.07495 \\
\hline $\begin{array}{l}\text { Days to } 50 \% \\
\text { Pods Setting }\end{array}$ & 0.3136 & 0.93214 & -0.79027 & -0.00286 & -0.00657 & -0.0998 & -0.04838 & -0.24551 & -0.07526 & 0.12048 & -0.05593 & -0.0642 & 0.08237 \\
\hline Days to maturity & 0.31191 & 0.93261 & -0.78988 & -0.00283 & -0.00907 & -0.09508 & -0.046 & -0.24046 & -0.0698 & 0.11394 & -0.05908 & -0.06803 & 0.08577 \\
\hline $\begin{array}{l}\text { Plant height } \\
\text { (cm) }\end{array}$ & 0.0851 & -0.14841 & 0.12482 & 0.01793 & -0.03109 & 0.05926 & 0.04675 & 0.05784 & 0.0346 & -0.15363 & -0.08198 & 0.24944 & -0.18469 \\
\hline $\begin{array}{c}\text { Number of } \\
\text { branches/plant }\end{array}$ & 0.02154 & -0.10088 & 0.1179 & -0.00918 & 0.06075 & -0.08931 & -0.14223 & -0.36564 & -0.00963 & 0.08906 & 0.1284 & -0.11834 & -0.03674 \\
\hline $\begin{array}{c}\text { Number of } \\
\text { clusters/plant }\end{array}$ & -0.12852 & -0.23222 & 0.18747 & 0.00265 & -0.01354 & 0.40059 & 0.1448 & 0.18331 & 0.01893 & -0.06147 & -0.06578 & 0.00308 & 0.38027 \\
\hline $\begin{array}{l}\text { Number of } \\
\text { pods/plant }\end{array}$ & -0.07587 & -0.13987 & 0.11268 & 0.0026 & -0.0268 & 0.17989 & 0.32245 & 0.15789 & 0.01776 & -0.03439 & -0.10595 & 0.09352 & 0.24592 \\
\hline $\begin{array}{l}\text { Number of } \\
\text { pods/cluster }\end{array}$ & -0.27741 & -0.56801 & 0.47143 & 0.00257 & -0.05513 & 0.18227 & 0.12637 & 0.4029 & 0.08332 & -0.23004 & -0.05319 & 0.05346 & 0.01719 \\
\hline $\begin{array}{l}\text { Number of } \\
\text { seeds/pod }\end{array}$ & 0.20082 & 0.52371 & -0.41156 & -0.00463 & 0.00437 & -0.0566 & -0.04276 & -0.2506 & -0.13396 & 0.18309 & -0.02961 & -0.07144 & 0.13584 \\
\hline Pod length $(\mathrm{cm})$ & -0.03363 & 0.33862 & -0.27136 & -0.00831 & 0.01631 & -0.07424 & -0.03343 & -0.27945 & -0.07395 & 0.33166 & -0.01245 & -0.10191 & 0.16759 \\
\hline $\begin{array}{c}100 \text { seed weight } \\
\text { (g) }\end{array}$ & -0.07265 & -0.2158 & 0.19317 & -0.00609 & 0.03229 & -0.10908 & -0.14142 & -0.08872 & 0.01642 & -0.0171 & 0.24158 & -0.16831 & -0.05144 \\
\hline $\begin{array}{c}\text { Biological } \\
\text { yield/plant (g) }\end{array}$ & 0.05823 & -0.20238 & 0.18172 & 0.01513 & -0.02431 & 0.00417 & 0.10198 & 0.07284 & 0.03237 & -0.1143 & -0.13751 & 0.29569 & -0.21164 \\
\hline $\begin{array}{c}\text { Harvest index } \\
(\%)\end{array}$ & -0.05682 & 0.14665 & -0.12939 & -0.00633 & -0.00426 & 0.29095 & 0.15145 & 0.01322 & -0.03476 & 0.10616 & -0.02374 & -0.11953 & 0.52357 \\
\hline
\end{tabular}

Residual Effect $=0.07888$ 
Table.5 Direct and indirect effects between yield and its related traits in 23 Black gram genotypes at phenotypic level

\begin{tabular}{|c|c|c|c|c|c|c|c|c|c|c|c|c|c|}
\hline Characters & DF50 & DP50 & DM & PH & PB & CPP & PPP & PPC & SPP & PL & HSW & BY & HI \\
\hline $\begin{array}{l}\text { Days to } 50 \% \\
\text { flowering }\end{array}$ & -0.00138 & -0.20086 & 0.3002 & 0.02565 & 0.00054 & -0.00251 & -0.02249 & -0.07492 & -0.03058 & 0.00054 & -0.00488 & 0.02292 & -0.08566 \\
\hline $\begin{array}{l}\text { Days to } 50 \% \\
\text { Pods Setting }\end{array}$ & -0.00111 & -0.24845 & 0.37358 & -0.01629 & -0.00445 & -0.00195 & -0.01726 & -0.07099 & -0.03537 & 0.0149 & -0.0062 & -0.03785 & 0.10894 \\
\hline Days to maturity & -0.0011 & -0.24677 & 0.37612 & -0.01633 & -0.00491 & -0.0018 & -0.0152 & -0.06629 & -0.03412 & 0.01458 & -0.00628 & -0.03862 & 0.11183 \\
\hline $\begin{array}{l}\text { Plant height } \\
\text { (cm) }\end{array}$ & -0.00028 & 0.0323 & -0.04903 & 0.12532 & -0.01777 & 0.00127 & 0.02214 & 0.01228 & 0.01913 & -0.01479 & -0.0105 & 0.16252 & -0.23492 \\
\hline $\begin{array}{c}\text { Number of } \\
\text { branches/plant }\end{array}$ & -0.00001 & 0.01492 & -0.02492 & -0.03002 & 0.07418 & -0.00058 & -0.02335 & -0.04407 & -0.02139 & -0.00139 & 0.01112 & -0.04485 & 0.03302 \\
\hline $\begin{array}{c}\text { Number of } \\
\text { clusters/plant }\end{array}$ & 0.00037 & 0.05175 & -0.07231 & 0.01693 & -0.00458 & 0.00936 & 0.08339 & 0.06278 & 0.00814 & -0.00531 & -0.00792 & -0.00863 & 0.53126 \\
\hline $\begin{array}{l}\text { Number of } \\
\text { pods/plant }\end{array}$ & 0.00018 & 0.02451 & -0.03267 & 0.01586 & -0.0099 & 0.00446 & 0.17496 & 0.07802 & 0.00406 & -0.00104 & -0.01094 & 0.04453 & 0.4273 \\
\hline $\begin{array}{l}\text { Number of } \\
\text { pods/cluster }\end{array}$ & 0.00053 & 0.08985 & -0.12701 & 0.00784 & -0.01665 & 0.00299 & 0.06954 & 0.1963 & 0.02172 & -0.0174 & -0.002 & 0.00747 & 0.10434 \\
\hline $\begin{array}{l}\text { Number of } \\
\text { seeds/pod }\end{array}$ & -0.00039 & -0.08193 & 0.11962 & -0.02234 & 0.01479 & -0.00071 & -0.00662 & -0.03975 & -0.10728 & 0.0061 & -0.00239 & -0.03234 & 0.15036 \\
\hline Pod length $(\mathrm{cm})$ & -0.00001 & -0.06766 & 0.1002 & -0.03388 & -0.00188 & -0.00091 & -0.00332 & -0.06243 & -0.01196 & 0.05472 & -0.00269 & -0.04792 & 0.12401 \\
\hline $\begin{array}{c}100 \text { seed weight } \\
\text { (g) }\end{array}$ & 0.0002 & 0.04561 & -0.06993 & -0.03893 & 0.02442 & -0.00219 & -0.05665 & -0.01163 & 0.00758 & -0.00435 & 0.03379 & -0.10309 & -0.02319 \\
\hline $\begin{array}{c}\text { Biological } \\
\text { yield/plant (g) }\end{array}$ & -0.00014 & 0.0426 & -0.06581 & 0.09228 & -0.01507 & -0.00037 & 0.0353 & 0.00665 & 0.01572 & -0.01188 & -0.01578 & 0.22071 & -0.30156 \\
\hline $\begin{array}{c}\text { Harvest index } \\
(\%)\end{array}$ & 0.00015 & -0.03456 & 0.05371 & -0.0376 & 0.00313 & 0.00635 & 0.09547 & 0.02616 & -0.0206 & 0.00866 & -0.001 & -0.085 & 0.78306 \\
\hline
\end{tabular}

Residual Effect $=0.16582$ 
Correlation association studies revealed that the characters number of clusters per plant, number of pods per plant, number of pods per cluster, number of seeds per pod and harvest index showed significant and positive correlation with seed yield per plant indicating that selection based on these characters would bring about simultaneous improvement in the seed yield.

Path analysis revealed that number of clusters per plant, number of pods per plant, number of pods per cluster and harvest index had true relationship by establishing significant positive associations and positive direct effects on seed yield per plant. Considering the nature and magnitude of character association and their direct and indirect effects, it can be inferred that improvement of seed yield per plant is possible through simultaneous selection of these traits. The residual effect permits precise explanation about the pattern of interaction of other possible components of yield and in the present study, the residual effect is 0.07888 indicating that the characters included in present investigation had contributed around 93 per cent of variability pertaining to the dependent variable i.e., seed yield per plant.

\section{References}

Al-Jibouri, H. A., Muller, P. A., and Robinson, H. F. (1958). Genetic and environmental variances and covariances in an upland cotton cross of inter specific origin. Journal of Agronomy, 50: 633-636.

Arya. P., Lal, G.M and Lal. S.S. (2017). Correlation and path analysis for yield and yield components in blackgram (Vigna mungo). International Journal of Advanced Biological Research. 7(2): 382-386.

Burton, G.W. (1952). Quantitative inheritance in grass. Proceedings of $6 \mathrm{th}$
International Grassland Congress, Pennsylvania State College, U.S.I: $277-$ 283.

Burton, G. W. and De Vane, E. M. (1953). Estimating heritability in tall fesses from replicated cloned material. Journal of Agronomy, 45 (3): 474-481.

Chauhan, M.P., Mishra, A.C and Singh, A.K. (2007). Correlation and path analysis in urdbean. Legume Research. 30(3): 205208.

Dewey, D.R and Lu, K.H. (1959). A correlation path coefficient analysis of components of crested wheat grass. Agronomy Journal. 51: 515-518.

Falconer, D. S. (1964). Introduction to quantitative genetics. Ronald Press, New York.

Fisher, R.A and Yates, F. (1967). Statistical tables for biological, agricultural and medical research. Oliver and Boyd Ltd., Edinburgh. 46-63.

Johnson, H.W., Robinson, H.O and Comstock, R.E. (1955). Estimates of genetic and environmental variability in soybean. Agronomy Journal. 47: 314318.

Kanimoli M. M, Shunmugavalli N, Muthuswamy A and Vijulan Harris C. (2015). Correlation and path analysis in blackgram (Vigna mungo (L.) Hepper). Agricultural Science Digest, 35(2): 158160.

Kasundra, J.K., Pethani, K.V and Kathiria, K.B. (1995). Studies on genetic variability, correlation and path analysis in urdbean. Indian Journal of Pulses Research.8(2): 113-118.

Kumar, G.V., Vanaja, M., Vagheera, P and Jyothi Laxmi, N. (2015). Correlation analysis for qualitative traits in blackgram (Vigna mungo (L.) Hepper) in different seasons. International Journal of Scientific and Research Publications. 5(4). 1-10.

Lush, J.L. (1940). Intra-sire correlation and 
regression of offspring in rams as a method of estimating heritability of characters. Proceedings of American Society of Animal Production. 33: 292301.

Patel, R.V., Patil, S.S., Patel, S.R and Jadhav, B.D., (2014). Genetic Variability and Character Association in Blackgram [Vigna mungo (L.) Hepper] during summer. Trends in Biosciences; 7(23): 3795-3798.

Panse, V.G and Sukhatme, P.V. 1961. Statistical Method for Research Workers. 2nd edition. ICAR, New Delhi, 351.

Patidar, M., Hemlata, Sharma. Haritwal and Santra. (2018). Genetic variability studies in blackgram [Vigna mungo (L.) Hepper]. International Journal of Chemical Studies;6(2): 1501-1503.

Rahim, M.A., Mia, A.A., Mahmud, F., Zeba $\mathrm{N}$ and Afrin, K.S.(2010). Genetic variability, character association and genetic divergence in mungbean (Vigna radiate L. Wilczek). Plant Omics Journal. 3(1): 1-6.

Reddy, D.K.R., Venkateswarlu, O., Siva Jyothi, G.L and Obaiah, M.C.(2011). Genetic parameters and interrelationship analysis in blackgram [Vigna mungo (L.) Hepper]. Legume Research. 34: 149-152.

Reddy, Y.V.K., Lal, S.S and Gabrial, M.L. (2017). Studies on genetic diversity in blackgram (Vigna mungo L. Hepper) germplasm. Global Journal of Bio-
Science and Technology;6(2): 296-302.

Rolania, D.K., Jinjwadia, M.K., Meghawal, D.R and Lal, G.M. (2017). Studies on genetic variability in black gram (Vigna mungo L. Hepper) germplasm. Journal of Pharmacognosy and Phytochemistry;6(4): 1506-1508.

Sharma, D.K., Billore, $M$ and Shrivastava, M.(2006). Estimation of variability parameters in black-gram (Vigna mungo L.) in Western Madhya Pradesh. Biosciences, Biotechnology Research Asia. 3(1a): 283-284.

Sivasubramanian, $\mathrm{P}$ and Madhavamenon, $\mathrm{P}$. 1973. Genotypic and phenotypic variability in rice. Madras Agricultural Journal. 60: 1093-1096.

Shalini Ch and GM Lal (2019) Correlation and path coefficient analysis for yield and yield components in blackgram (Vigna mungo (L.) Hepper). The Pharma Innovation Journal; 8(6): 6568.

Umadevi, M and Ganesan, N.M. (2005). Correlation and path analysis for yield and yield components in blackgram [Vigna mungo (L.) Hepper]. Madras Agriculture Journal; 92(10-12): 731734.

Usharani, K. S. and Anandakumar, C. R. (2015). Ems induced mutations in urdbean (Vigna mungo (L.) Hepper) and significance of induced altered correlations. Life Sciences Leaflets; 63: 140-146.

\section{How to cite this article:}

Alle N. Sarath, C. Reddy, Gabrial M. Lal and Siva Krishna, V. 2020. Study of Quantitative Traits and Association for Yield and its Components in Black Gram (Vigna mungo (L.) Hepper) Genotypes. Int.J.Curr.Microbiol.App.Sci. 9(09): 1943-1952. doi: https://doi.org/10.20546/ijcmas.2020.909.244 